\title{
Résumé de thèse. La conception du travail en horaires atypiques : une démarche d'innovation sociale conduite en milieu hospitalier
}

\section{Marlène Cheyrouze}

\section{OpenEdition}

Journals

Édition électronique

URL : https://journals.openedition.org/activites/7108

DOI : 10.4000/activites.7108

ISSN : 1765-2723

Éditeur

ARPACT - Association Recherches et Pratiques sur les ACTivités

Référence électronique

Marlène Cheyrouze, « Résumé de thèse. La conception du travail en horaires atypiques

une démarche d'innovation sociale conduite en milieu hospitalier », Activités [En ligne], 18-2| 2021, mis en ligne le 15 octobre 2021, consulté le 07 avril 2022. URL : http://journals.openedition.org/activites/ 7108 ; DOI : https://doi.org/10.4000/activites.7108

Ce document a été généré automatiquement le 7 avril 2022.

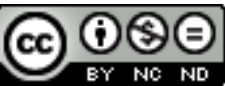

Activités est mis à disposition selon les termes de la licence Creative Commons Attribution - Pas d'Utilisation Commerciale - Pas de Modification 4.0 International. 


\title{
Résumé de thèse. La conception du travail en horaires atypiques : une démarche d'innovation sociale conduite en milieu hospitalier
}

\author{
Marlène Cheyrouze
}

\section{RÉFÉRENCE}

Marlène Cheyrouze (2021). "La conception du travail en horaires atypiques :

une démarche d'innovation sociale conduite en milieu hospitalier", sous la direction de Béatrice Barthe. Soutenue le 25 juin 2021

\section{Contexte}

1 Cette thèse porte sur la conception du travail en horaires atypiques et s'inscrit dans une recherche-action. Le terrain est une clinique privée qui, face à l'augmentation de l'absentéisme et du turn over des soignants, recherche une organisation temporelle de travail innovante. En ergonomie, la conception du temps de travail ne se limite pas au changement des horaires et s'accompagne d'une véritable réflexion sur la nature et les conditions du travail concerné (Barthe, Gadbois, Prunier-Poulmaire, \& Quéinnec, 2004 ; Toupin, Barthe, \& Prunier-Poulmaire, 2013). C'est la situation de travail entière qu'il s'agit de concevoir de façon participative avec les salariés, pour choisir le compromis le plus acceptable (Quéinnec, Teiger, \& de Tersssac, 2008). Selon un courant scientifique ancré dans les sciences sociales, économiques, politiques et gestionnaires (Cloutier, 2003 ; Lapointe, Bellemare, Briand, D'Amours, Grant, Laplante et al., 2007), la conception du temps de travail peut être considérée comme une innovation sociale pour ses effets positifs sur la conciliation entre les vies professionnelle et privée. L'innovation sociale 
peut se situer dans de nouveaux horaires (ex. horaires flexibles, temps partiel), c'est-àdire dans les solutions retenues, mais aussi dans leur processus de création. Deux conditions sont nécessaires : les acteurs concernés participent au choix des solutions pour se les approprier et leur donner du sens; leurs logiques sont différentes pour créer des coopérations inédites et construire une représentation partagée des problèmes et des solutions possibles (Tremblay, 2014).

\section{Thèse défendue}

2 La conception de nouveaux horaires est une opportunité d'engager la diversité des acteurs dans la discussion des conditions et des règles du travail concerné. Bien que les changements d'horaires aient des répercussions positives sur les travailleurs, nous considérons que la conception du temps de travail - telle qu'elle est portée par l'ergonomie - est une innovation sociale du point de vue de sa démarche, plutôt que du point de vue des solutions.

\section{Problématique}

3 Pour produire des connaissances sur la conception du travail en horaires atypiques et démontrer que la démarche de l'ergonomie peut être une démarche d'innovation sociale, 5 intentions de recherche sont visées.

4 Notre revue de la littérature sur l'absentéisme et le turnover des soignants montre qu'ils sont aussi bien déterminés par les contraintes d'horaires que par les contraintes du travail et les interactions professionnelles. Du point de vue de l'ergonomie, le travail a des contraintes (physiques, cognitives, émotionnelles, temporelles) qui peuvent augmenter la pénibilité des horaires. L'approche multifactorielle et systémique des temps de travail (Anses, 2016 ; Barthe, 2016) peut être un guide pertinent pour analyser la situation initiale. Notre $1^{\text {ère }}$ intention est de confirmer que les points de tension qui seront identifiés dans le diagnostic se situent aux niveaux des horaires, des contraintes du travail et des interactions professionnelles.

5 La conception du temps de travail ne saurait se passer d'une réflexion sur la nature et les conditions du travail lui-même (Barthe et al., 2004; Toupin et al., 2013). Pour les différents acteurs qui y participent, c'est une occasion de discuter les règles de l'organisation «à froid» (de Terssac \& Lompré, 1996) et de découvrir les différentes représentations sur le travail (Petit \& Dugué, 2013). L'animation du dispositif peut reposer sur des objets intermédiaires, pour incarner une base de travail commune (Jeantet, 1998) et concrétiser les discussions (Caroly \& Barcellini, 2013). Notre $2^{\mathrm{e}}$ intention est de montrer que l'animation d'un dispositif participatif rassemblant une diversité de logiques professionnelles et se basant sur des supports intermédiaires fournit un cadre pour accéder aux réalités du travail actuel de chacun.

6 La simulation organisationnelle semble être une voie méthodologique pertinente pour mettre des scénarios d'organisation temporelle à l'épreuve de tous les facteurs impliqués et pour co-construire les conditions de réalisation de l'activité future (Van Belleghem \& Barthe 2016). Notre $3^{\mathrm{e}}$ intention est de montrer que l'animation d'un dispositif participatif inspiré de la simulation organisationnelle peut aider les acteurs à co-construire les conditions d'application des solutions, à anticiper leurs effets sur les 
facteurs du travail et du hors-travail, et à co-construire des solutions riches et pertinentes.

7 La simulation peut aider les travailleurs à s'approprier les changements et à leur donner du sens (Bobillier Chaumon, Rouat, Laneyrie, \& Cuvillier, 2018). Notre $4^{\mathrm{e}}$ intention est de vérifier que les changements d'horaires, co-construits dans le dispositif et expérimentés en situation réelle, ont des répercussions positives sur les facteurs du travail et du hors-travail.

En milieu hospitalier, plusieurs logiques professionnelles doivent se coordonner et celles des médecins sont dominantes (Raveyre \& Ughetto, 2003). Leur engagement dans la démarche est incontournable et détermine fortement les possibilités de transformation. Notre $5^{\mathrm{e}}$ intention est de vérifier que les solutions co-construites avec les médecins se concrétisent en situation réelle, et de montrer que leurs conditions de réussite peuvent devenir de nouvelles règles.

\section{Méthode}

Pour répondre à ces intentions de recherche, notre démarche de conception a été conduite dans un service de pneumologie selon quatre phases.

Le diagnostic de la situation initiale (phase 1) a été guidé par l'approche multifactorielle et systémique des temps de travail et s'est basé sur plusieurs techniques de recueil: une consultation des fiches de poste, une récolte de données chiffrées sur les entrées et sorties de patients, 21 recueils d'observations (ouvertes et systémiques), 21 entretiens (autoconfrontations; semi-directifs; non directifs) et 15 questionnaires. Il s'agissait d'identifier les points de tension saillants, et de formuler des principes de solutions en accord avec les données du terrain et de la littérature.

11 La construction du diagnostic partagé (phase 2) et la projection des solutions (phase 3) se sont basées sur l'animation de "groupes-terrains » et de " groupes-décisionnaires ». Les fonctions des groupes-terrains étaient de construire et projeter les solutions. Les participants pouvaient être des soignants (infirmières, aides-soignants, hôtelières), des médecins, des cadres et des secrétaires. Les fonctions des groupes-décisionnaires étaient de valider ou non la poursuite de la démarche pour chaque solution. Les participants pouvaient être des soignants, des médecins, des cadres, des membres de la Direction et des délégués du personnel. La projection des solutions (phase 3) s'est basée sur l'animation de 8 groupes-terrains et 5 groupes-décisionnaires. Des supports intermédiaires inspirés de la simulation organisationnelle ont été utilisés dans tous les groupes-terrains, notamment des maquettes «spatiales » (représentations du service à échelle réduite), «temporelles» (frises chronologiques) et "cognitives" (représentations de raisonnements associés aux solutions projetées). Les échanges ont été enregistrés et retranscrits. Les 5 groupes-terrains réunissant plus de deux logiques professionnelles différentes ont été codés sur Actograph ${ }^{\circledast}$ pour relever les verbalisations du travail actuel, et ont fait l'objet d'une analyse thématique de contenu pour relever les croisements de points de vue. Tous les groupes-terrains ont fait l'objet d'une autre analyse thématique de contenu pour relever les conditions de réussite et les effets des solutions, verbalisés par les participants. Toutes les données indiquant que les solutions étaient validées, réajustées, sous conditions ou éliminées ont été extraites des retranscriptions, des supports, des notes et des comptes rendus de chaque 
séance. Leur analyse a permis de reconstituer le cheminement des solutions sur le logiciel Actograph ${ }^{\oplus}$.

12 Les solutions retenues ont été testées et évaluées pendant trois mois (phase 4), avec plusieurs techniques de recueil: une récolte de données chiffrées sur les horaires de sortie dans le contrôle de gestion, une grille d'observation sur l'organisation des sorties, des entretiens rétrospectifs avec les soignants et des questionnaires. Il s'agissait d'évaluer la concrétisation des solutions, leurs répercussions, et l'ensemble de la démarche.

\section{Résultats}

13 Le diagnostic de la situation initiale (phase 1) confirme que les points de tension identifiés sont dans les trois déterminants de l'absentéisme et du turnover en milieu hospitalier. Dans les contraintes d'horaires : la conciliation entre le travail et le horstravail est freinée par le roulement et la politique des congés. Dans les contraintes du travail: le flux de patients (entrées; sorties) est imprévisible et sous pression temporelle; ses marges de régulation sont restreintes par les temporalités aléatoires des médecins. Dans les interactions professionnelles : infirmières et médecins ont des difficultés à se coordonner et il y a un débordement des horaires prescrits pour rallonger le chevauchement dédié aux transmissions orales.

Dans la construction du diagnostic partagé (phase 2), 14 solutions ont été proposées aux acteurs et 4 d'entre elles ont été progressivement éliminées de la démarche. La projection des 10 solutions restantes (phase 3 ) confirme que des contraintes et des règles du travail actuel ont été verbalisées dans tous les groupes-terrains rassemblant plus de deux logiques professionnelles différentes. Des croisements de points de vue ont été relevés dans trois groupes-terrains : entre les soignants et les cadres, ou entre les soignants et les médecins. Les soignants ont saisi plusieurs groupes-terrains pour confronter les propositions de la cadre à la réalité de leur travail, ou pour interpeller les cadres et les médecins sur des problématiques existantes. Des maquettes ont aidé les participants à projeter les effets de changements d'horaires sur des facteurs du travail (ex. avancer le dernier tour de soins) et du hors-travail (ex. manquer le premier bus du matin). Les participants ont posé les conditions de réussite pour tous les changements d'horaires, dont plusieurs relatives à l'organisation du travail lui-même. Par exemple : l'avancée du poste de l'infirmière de jour, destinée à rallonger le chevauchement journuit, était conditionnée par une meilleure anticipation du flux de patients et des prescriptions médicales. Parmi les 10 solutions projetées, 3 ont été progressivement éliminées de la démarche.

L'expérimentation et l'évaluation des 7 solutions restantes (phase 4) révèlent que les changements d'horaires des infirmières ont eu des effets positifs sur le travail (ex. gain de temps pour les premières tâches de la journée) et sur le hors-travail (ex. avancée du retour au domicile). Ces changements reposaient sur des conditions co-construites avec les médecins dans les groupes-terrains. D'après les données du contrôle de gestion, de la grille d'observation et des entretiens rétrospectifs, plusieurs de ces conditions se sont concrétisées dans le service pour devenir de nouvelles règles. Par exemple: les infirmières se sont mises à téléphoner aux médecins pour les prévenir de l'arrivée des entrées, ou les médecins se sont mis à anticiper la sortie des patients pour qu'ils partent plus tôt. D'après les entretiens, la démarche a permis d'ouvrir un dialogue avec 
les médecins sur les problématiques existantes, d'accéder aux représentations particulières du travail de chacun, et d'en diminuer les contraintes. En définitive, la participation des médecins, et les collaborations inédites qu'elle a occasionnées ont été davantage valorisées par les soignants que le changement d'horaires lui-même.

\section{Discussion}

16 Les apports de cette thèse sont de plusieurs niveaux. À un niveau théorique, nous proposons de considérer la conception du temps de travail - telle qu'elle est portée par l'ergonomie - comme une démarche d'innovation sociale, capable d'aboutir au choix d'horaires le plus acceptable et de réduire simultanément les contraintes du travail concerné. Dans la lignée des travaux sur les innovations sociales, l'ergonomie peut en enrichir les concepts dans le champ particulier du travail et de l'entreprise. Nous proposons également de compléter l'approche multifactorielle et systémique des temps de travail avec un autre facteur: l'interdépendance des acteurs qui composent le collectif de travail.

17 À un niveau méthodologique, outiller une démarche de conception participative avec des supports inspirés de la simulation organisationnelle permet d'encadrer les échanges de points de vue sur la réalité du travail, de confronter celle-ci aux solutions proposées, et d'en co-construire les conditions de réussite pour mieux se les approprier. Dans les interventions à venir, le couplage de l'approche multifactorielle et systémique des temps de travail avec la simulation organisationnelle semble prometteur. Nous proposons également d'actualiser la démarche établie par Quéinnec et al. (2008), en préconisant de baser la recherche du compromis le plus acceptable sur l'articulation de groupes-décisionnaires et de groupes-terrains auxquels participent les acteurs de pouvoir, et en préconisant d'expérimenter et d'évaluer les solutions retenues en situation réelle et sur une période définie.

À un niveau politique et sachant que «la culture de l'innovation » est aujourd'hui un levier de compétitivité pour les entreprises, de nouvelles perspectives peuvent s'ouvrir à l'ergonomie dans la conception de démarches innovantes, destinées à développer des organisations innovantes. La perspective est d'autant plus intéressante pour le milieu hospitalier, dont les conditions de travail sont dans un état préoccupant, et que la crise sanitaire a mis en relief. Pour pérenniser le dispositif déployé dans cette rechercheaction, nous préconisons de soutenir la participation des personnels hospitaliers par des moyens matériels, humains et financiers (réservation de salles, rémunération du temps investi, prévision du remplacement dans le service). Nous préconisons aussi d'intégrer systématiquement les médecins dans les réflexions sur l'organisation et les réalités du travail. Enfin, nous préconisons de restituer aux cadres de proximité le temps nécessaire à la construction et l'animation du dispositif. 


\section{BIBLIOGRAPHIE}

Agence nationale de sécurité sanitaire de l'alimentation, de l'environnement et du travail. (2016). Évaluation des risques sanitaires liés au travail de nuit. Rapport d'expertise collective.

Barthe, B. (2016). Temps de travail atypiques : Désaccords temporels, des accords par l'activité: Perspectives individuelles, collectives et socio-familiales pour aménager les temps de travail. [Habilitation à diriger des recherches], Université Toulouse.

Barthe, B., Gadbois, C., Prunier-Poulmaire, S., \& Quéinnec, Y. (2004). Travailler en horaires atypiques. In P. Falzon, Ergonomie (p. 129-144). PUF.

Bobillier Chaumon, M.-É., Rouat, S., Laneyrie, E., \& Cuvillier, B. (2018). De l'activité DE simulation à l'activité EN simulation : Simuler pour stimuler. Activités, 15(1).

Caroly, S., \& Barcellini, F. (2013). Le développement de l'activité collective. In P. Falzon, Ergonomie constructive (p. 33-46). Paris : PUF.

Cloutier, J. (2003). Qu'est-ce que l'innovation sociale ? ( ${ }^{\circ}$ ET0314; Études théoriques). CRISES.

Jeantet, A. (1998). Les objets intermédiaires dans la conception. Éléments pour une sociologie des processus de conception. Sociologie du travail, 40(3), 291-316.

Lapointe, P.-A., Bellemare, G., Briand, L., D’Amours, M., Grant, M., Laplante, N., Legault, M.-J., Lévesque, B., Paquet, R., Bélanger, P. R., Bernier, C., Cucumel, G., Gislain, J.-J., Harrisson, D., Legault, G., Lessemann, F., Malenfant, R., \& Tremblay, D.-G. (2007). Axe 1 - Travail et emploi. In J.-L. Klein \& D. Harrison, L'innovation sociale : Émergence et effets sur la transformation des sociétés (p. 345-360). Presses de l'Université du Québec.

Quéinnec, Y., Teiger, C., \& de Terssac, G. (2008). Repères pour négocier le travail posté. Toulouse : Octarès Éditions.

Raveyre, M., \& Ughetto, P. (2003). Le travail, la part oubliée des restructurations hospitalières. Revue française des affaires sociales, 1(3), 95-119.

Terssac (de), G., \& Lompré, N. (1996). Pratiques organisationnelles dans les ensembles productifs : Essai d'interprétation. In J.-C. Sperandio, L'ergonomie face aux changements technologiques et organisationnels du travail humain (p. 51-66). Toulouse : Octarès Éditions.

Toupin, C., Barthe, B., \& Prunier-Poulmaire, S. (2013). Du temps contraint au temps construit : Vers une organisation capacitante du travail en horaires alternants et de nuit. In P. Falzon, Ergonomie constructive (p. 75-88). Paris : PUF.

Tremblay, D.-G. (2014). L'innovation technologique, organisationnelle et sociale. Presses de l'Université du Québec.

Van Belleghem, L. V., \& Barthe, B. (2016). Temps de travail, temps de vivre : Vers une conciliation des vies professionnelle et privée. In M. Christol-Souviron, A. Drouin, \& P. Étienne, «Performances Humaines et Techniques ", d'hier vers aujourd'hui. (p. 521-528). Toulouse : Octarès Éditions.

\section{RÉSUMÉS}

La conception des temps de travail peut être considérée comme une innovation sociale dans les solutions retenues et dans leur processus de création. Portant le point de vue de l'ergonomie, 
cette thèse propose une démarche de conception du travail en horaires atypiques. Conduite en milieu hospitalier, cette démarche est une opportunité d'engager la diversité des acteurs dans une réflexion collective sur les conditions et les règles du travail concerné.

INDEX

Mots-clés : horaires atypiques, conception, organisation, hôpital, innovation, temporalités

\section{AUTEURS}

MARLÈNE CHEYROUZE

Laboratoire CLLE. Université Toulouse Jean Jaurès - marlene.cheyrouze@gmail.com 